\title{
FACTORS OF INFINITE-DIMENSIONAL MANIFOLDS
}

\author{
BY \\ R. D. ANDERSON AND R. SCHORI( ${ }^{(1)}$
}

1. Introduction. Let $J^{\infty}$ denote the Hilbert cube, i.e. the countable infinite product of closed intervals, and let $s$ denote the countable infinite product of open intervals (or lines as convenient). Specifically, let $J^{\infty}=\prod_{i>0} J_{i}$ and let $s=\prod_{i>0} J_{i}^{0}$ where for each $i>0, J_{i}$ is the closed interval $[-1,1]$ and $J_{i}^{0}$ is the open interval $(-1,1)$. In [1] it was shown that $s$ is homeomorphic to Hilbert space, $l_{2}$, and thus, on the basis of results in [6] and [7], to all separable infinite-dimensional Fréchet spaces (and therefore, of course, to all separable infinite-dimensional Banach spaces).

A Fréchet manifold (or F-manifold) is a separable metric space with an open cover of sets each homeomorphic to an open subset of $s$. Thus all separable metric Banach manifolds modeled on separable infinite-dimensional Banach spaces are $F$-manifolds. A Fréchet manifold is known to admit a complete metric and to be nowhere locally compact.

A Hilbert cube manifold or Q-manifold is a separable metric space with an open cover of sets homeomorphic to open subsets of $J^{\infty}$. A $Q$-manifold is known to admit a complete metric and to be locally compact. We could, equivalently, specify that a $Q$-rhanifold admits an open cover by sets whose closures are homeomorphic to $J^{\infty}$ itself.

The following are the principle theorems of this paper.

THEOREM I. If $M$ is any F-manifold, then $s \times M$ is homeomorphic to $M$. (For a somewhat stronger version of this theorem see the addendum at the end of this paper.)

THEOREM II. If $M$ is any $Q$-manifold, then $J^{\infty} \times M$ is homeomorphic to $M$.

Since $s$ is known [2] or [5] to be homeomorphic to $s \times J^{\infty}$, from Theorem I we immediately have the following.

Corollary. If $M$ is any F-manifold, then $J^{\infty} \times M$ is homeomorphic to $M$.

Almost identical proofs of Theorems I and II can be given. We shall explicitly give the proof of Theorem I only. It will be understood that the proof of Theorem I also constitutes a proof of Theorem II with the natural modifications needed such as $s$ replaced by $J^{\infty}, F$-manifold replaced by $Q$-manifold and open interval factors of $s$ replaced by closed interval factors of $J^{\infty}$.

Presented to the Society, August 29, 1968; received by the editors August 5, 1968.

(1) The research leading to this paper was supported in part under NSF Grants GP-6867 and GP-8637. 
In the next section we shall reduce the proof of Theorem I to the proof of Lemma $B$.

2. Reduction of Theorem I to Lemma B. We begin with a definition.

Let $r$ be a continuous function of a topological space $X$ into the closed unit interval $I=[0,1]$. Let $J^{0}(0)=\{0\}$ and for $t \in(0,1]$, let $J^{0}(t)=(-t, t)$. Then

$$
J^{0} \times^{r} X=\left\{(y, x) \in J^{0} \times X: y \in J^{0}(r(x))\right\}
$$

is the variable product of $J^{0}$ by $X$ (with respect to $r$ ). Likewise, let $s_{0}$ be the origin of $s$ or, where convenient, the single point set consisting of the origin of $s$ and for $t \in(0,1]$, let $s_{t}=\prod_{i>0} J_{i}^{0}(t)$ where $J_{i}^{0}(t)=(-t, t)$. Note that for each $t, s_{t} \subset s$. Then

$$
s \times^{r} X=\left\{(y, x) \in s \times X: y \in s_{r(x)}\right\}
$$

is the variable product of $s$ by $X$ (with respect to $r$ ). If $A \subset X$, let $J^{0} \times{ }^{r} A$ (or $s \times^{r} A$ ) be the variable product of $J^{0}$ (or $s$ ) by $A$ (with respect to $r \mid A$ ).

We are now in a position to state Lemmas A and B.

LEMMA A. Let $M$ be an F-manifold, let $V \subset U \subset M$ where $V$ is closed and $U$ is open and is homeomorphic to an open subset of $s$, and let $s \times^{r_{0}} M$ be a variable product of $s$ by $M$. There exists a homeomorphism $H$ of $s \times{ }^{r_{0}} M$ onto a variable product $s \times{ }^{r} M$ such that (1) $r \leqq r_{0}$, (2) $r(V)=0$, and (3) $H \mid s \times^{r_{0}}\left[(M \mid U) \cup r_{0}^{-1}(0)\right]$ is the identity.

We now reformulate Lemma $\mathrm{A}$ in a somewhat more convenient form.

Lemma B. Let $U$ be an open subset of $s$, let $V \subset W \subset U$ where $W$ is open and $V$ is closed in $U$, and let $s x^{r_{0}} U$ be a variable product of $s$ by $U$. There exists a homeomorphism $H$ of $s \times r_{0} U$ onto a variable product $s \times{ }^{r} U$ such that (1) $r \leqq r_{0},(2) r(V)=0$, and (3) $H \mid s \times{ }^{r_{0}}\left[(U \backslash W) \cup r_{0}^{-1}(0)\right]$ is the identity.

Proof that Lemma B implies Lemma A. Letting $M, V, U$, and $s \times{ }^{r_{0}} M$ be as in the hypothesis of Lemma A, we may let $W$ be any open set in $M$ such that $V \subset W \subset U$ and the closure of $W$ in $M$ is the subset of $U$. Regarding such $U, V$, and $W$ as in the hypothesis of Lemma B and regarding $r_{0}$ in Lemma B as $r_{0} \mid U$, it follows that any homeomorphism $H$ as in the conclusion of Lemma $\mathrm{B}$ has an automatic extension to a homeomorphism satisfying the conditions of Lemma A.

Proof that Lemma A implies Theorem I.

Step 1. As proved in [8] and applied in [3], since $M$ is separable and metric, there exists a countable star-finite open cover $G$ of $M$ with sets homeomorphic to open subsets of $s$. (By a star-finite cover we shall mean a cover where the closure of each element of the cover intersects the closure of only finitely many elements of the cover.)

Step 2. We follow a procedure as used in Theorem 2 of [3]. Let $\left(g_{i}\right)_{i>0}$ be any ordering of the elements of $G$. Let $E_{1}=\left\{g_{1}\right\}$ and inductively let $E_{i+1}$ be the set containing the least indexed element $g_{k}$ which is not in $\bigcup_{j \leqq i} E_{i}$ together with all the elements of $G$ which are not in $\bigcup_{j \leqq i} E_{j}$ and which intersect some element of 
$E_{i}$. Clearly each $E_{i}$ must be finite. We now order the elements of $G$ as $\left(U_{i}\right)_{i>0}$ by first listing the element of $E_{1}$ and then inductively listing the elements of $E_{2 n+1}$ followed by those of $E_{2 n}$. It is easy to verify that for any sequence $\left(H_{i}\right)_{i>0}$ of homeomorphisms, it will follow that $\left(H_{i} \circ \ldots \circ H_{1}\right)_{i>0}$ converges to a homeomorphism of $s \times M$ onto $\bigcap_{i>0} H_{i} \circ \ldots \circ H_{1}(s \times M)$ provided (1) $H_{1}$ maps $s \times M$ into itself with $H_{1} \mid s \times\left(M \backslash U_{1}\right)=$ identity and (2) for each $i>0, H_{i+1}$ maps $H_{i} \circ \ldots$ $\circ H_{1}(s \times M)$ into itself with $H_{i+1} \mid\left[s \times\left(M \backslash U_{i+1}\right)\right] \cap H_{i} \circ \cdots \circ H_{1}(s \times M)=$ identity.

Step 3. We now apply Lemma A inductively to define $\left(H_{i}\right)_{i>0}$ as in Step 2 with $\bigcap_{i>0} H_{i} \circ \cdots \circ H_{1}(s \times M)=s_{0} \times M$ which is homeomorphic to $M$. For each $i>0$, let $V_{i} \subset U_{i}$ where $V_{i}$ is closed and $\left\{V_{i}\right\}_{i>0}$ covers $M$. By Lemma $A$ there exists a homeomorphism $H_{1}$ of $s \times M$ onto a variable product $s \times{ }^{r_{1}} M$ such that $r_{1}\left(V_{1}\right)=0$ and $H_{1} \mid s \times\left(M \mid U_{1}\right)=$ identity. Inductively, by Lemma $\mathrm{A}$ let $H_{i+1}$ be a homeomorphism of $s \times{ }^{r_{i}} M$ onto a variable product $s \times \times^{r_{i+1}} M$ where $r_{i+1} \leqq r_{i}, r_{i+1}\left(V_{i+1}\right)=0$, and $H_{i+1} \mid s \times{ }^{r_{i}}\left(M \backslash U_{i+1}\right)=$ identity. Hence, by Step 2 and by the definition of the $H_{i}$ we have $\left(H_{i} \circ \cdots \circ H_{1}\right)_{i>0}$ converging to a homeomorphism of $s \times M$ onto $s_{0} \times M$ which is homeomorphic to $M$.

3. Introduction to the proof of Lemma B. The proof of Lemma B will be given in $\S \S 3,4,5$, and 6 . It will be shown that the homeomorphism $H$ of Lemma B can effectively be defined on $s \times U \backslash r_{0}^{-1}(0)$ instead of on the variable product $s \times{ }^{r_{0}} U$. In fact, $H$ will ultimately be described by means of various interchanges of coordinates on $\left(y_{1}, y_{2}, \ldots, z_{1}, z_{2}, \ldots\right) \in s \times U \backslash r_{0}^{-1}(0)$ where $\left(y_{1}, y_{2}, \ldots\right) \in s$ and $\left(z_{1}, z_{2}, \ldots\right) \in U \backslash r_{0}^{-1}(0)$. In order that $H$ be continuous and a variable product we shall also shrink the coordinates in the $y_{i}$ coordinate places. We now become more explicit in our discussion.

Let $\left\{\alpha_{i}\right\}_{i}>0$ be a collection of disjoint infinite sets of integers whose union is the set of positive integers such that if the elements of each $\alpha_{i}$ are monotonically ordered as $(i, 1),(i, 2), \ldots$, then $(i, j)<(k, j)$ for $i<k$. The interchangings and shrinkings of coordinates are to occur only within the systems $\left(y_{i}, z_{(i, 1)}, z_{(i, 2)}, \ldots\right)$ but on all such systems simultaneously. Indeed, the interchanges and shrinkings to be described will be independent of $i$ and thus may be described simply by describing for any $i>0$ the procedure for $\left(y_{i}, z_{(i, 1)}, z_{(i, 2)}, \ldots\right)$. If $p=\left(y_{1}, y_{2}, \ldots, z_{1}, z_{2}, \ldots\right)$ $\in s \times s$, let $p^{i}=\left(y_{i}, z_{(i, 1)}, z_{(i, 2)}, \ldots\right)$ and for notational simplicity we shall refer to such a sequence as $\left(x_{0}, x_{1}, x_{2}, \ldots\right)$. Thus, $\left\{p^{i}: p \in s \times s\right\}=J_{i}^{0} \times \prod_{j>0} J_{(i, j)}^{0}$ and we will denote this by $X_{0}^{\infty}$. At our convenience we shall regard $X_{0}^{\infty}$ as $\prod_{j \geqq 0} X_{j}$ where $X_{0}=J_{i}^{0}$ and for $j>0, X_{j}=J_{(i, j)}^{0}$.

To analyze the types of coordinates of points $\left(y_{1}, y_{2}, \ldots, z_{1}, z_{2}, \ldots\right)$ in $s \times\left(U \backslash r_{0}^{-1}(0)\right)$ we note for $i>0$, that $y_{i}$ ranges over the interval $(-1,1)$ whereas the $z_{i}$ 's are restricted by the requirement that $\left(z_{1}, z_{2}, \ldots\right) \in U \backslash r_{0}^{-1}(0)$. However, since such a set is open in $s$, for a fixed point $\left(z_{1}, z_{2}, \ldots\right)$ in $U \backslash r_{0}^{-1}(0)$ there exists an integer $n$ such that $\left\{z_{1}\right\} \times \cdots \times\left\{z_{n}\right\} \times \prod_{i>n} J_{i}^{0} \subset U \backslash r_{0}^{-1}(0)$.

We now make some definitions. An open set $E$ of $s$ is an $n$-basic open set in $s$ 
if $E=E_{1} \times \cdots \times E_{n} \times \prod_{i>n} J_{i}^{0}$ where each $E_{i}$ is open in $J_{i}^{0}$ and is a subinterval but not necessarily a proper subinterval of $J_{i}^{0} . E$ is a basic open set in $s$ if $E$ is an $n$ basic open set in $s$ for some $n$. We correspondingly define an $n$-basic open set in $J^{\infty}$ by replacing $s$ with $J^{\infty}$ and $J_{i}^{0}$ with $J_{i}$ in the above. Thus, if $E$ is an $n$-basic open set in $s\left(\right.$ or $J^{\infty}$ ) and $m \geqq n$ then $E$ is also an $m$-basic open set in $s$ (or $J^{\infty}$ ). If $W$ is a subset of $s$, let $\pi: s \times W \rightarrow W$ be the natural projection onto $W$ and for $n>0$, let $\pi_{n}$ be defined on $W$ as follows. For $z=\left(z_{1}, z_{2}, \ldots\right) \in W$, let $\pi_{n}(z)=\left(z_{1}, \ldots, z_{n}\right.$, $0,0, \ldots)$. Note that in general $\pi_{n}$ does not map into $W$ but in our applications it usually does. Also, if $Y$ is a space and $f: W \rightarrow Y$ is a function, define $f^{*}: s \times W \rightarrow Y$ by $f^{*}=f \pi$.

Definition. Let $W$ be an open subset of $s$ and let $\left\{G_{i}\right\}$ be a star finite collection of $m_{i}$-basic open sets in $s$ (that is, for each $i, G_{i}$ is $m_{i}$-basic) whose union is $W$. For each $x \in W$, let

$$
m_{x}=\operatorname{minimum}\left\{m_{i}: x \in G_{i}\right\} .
$$

Let $Y$ be a topological space. A map, i.e. continuous function, $f: W \rightarrow Y$ is a local product map of $W$ with respect to the $G_{i}$ and $m_{i}$ if $f(x)=f\left(\pi_{m_{x}}(x)\right)$ for each $x \in W$. If, additionally, $Y=[1, \infty)$ and $f(x) \geqq m_{x}$ for each $x \in W$, then $f$ is a local product indicator map of $W$ with respect to the $G_{i}$ and $m_{i}$.

$A$ special case of Lemma B. We introduce, in a very special case, some of the procedures and notation to be used later. We shall assume the notation and conventions already introduced. For this special case of Lemma B we shall assume that for some $n>0$, (1) the open sets $U$ and $W$ are $n$-basic open sets in $s$, (2) $V$ is the closure of an $n$-basic open set in $s$, and (3) $r_{0}(x)=1$ for each $x \in U$. Observe that condition (3) implies that $s \times{ }^{r} 0 U=s \times U$. In the general case we shall not have this obvious product structure with respect to $U, W$, and $V$ and will have to identify suitable local product structures with respect to these sets and $r_{0}^{-1}(0)$. Now, consider the space $X_{0}^{\infty}$ of points $p^{i}=\left(y_{i}, z_{(i, 1)}, z_{(i, 2)}, \ldots\right)$ that have been relabeled $\left(x_{0}, x_{1}, x_{2}, \ldots\right)$. If the procedure to be described for interchanging coordinates in $X_{0}^{\infty}$ leaves the $x_{1}, \ldots, x_{n}$ coordinates of points in $X_{0}^{\infty}$ fixed, then the induced function will carry each point of $s \times U$ to a point of $s \times U$.

The desired homeomorphism $H$ of $s \times U$ onto $s \times{ }^{r} U$ will be expressed in terms of a map $h$ of $X_{0}^{\infty} \times I \times\{n\}$ into $X_{0}^{\infty}$ (with $\{n\}$ a single point set for our special case). The map $h$ is to be an isotopy such that for $x=\left(x_{0}, x_{1}, \ldots\right) \in X_{0}^{\infty}$ we have $h(x, 0, n)$ $=x$ and

$$
h(x, 1, n)=\left(0, x_{1}, \ldots, x_{n},-x_{0},-x_{n+1},-x_{n+2}, \ldots\right) .
$$

To finish the description of $h$ we first describe a map $h^{\prime}$ of $X_{0}^{\infty} \times[0,1) \times\{n\}$ into $X_{0}^{\infty}$ and then modify $h^{\prime}$ so as to produce $h$. Let $h^{\prime}(x, 0, n)=x$ and for each integer $i>0$, let

$$
h^{\prime}\left(x, 1-2^{-i}, n\right)=\left(x_{n+i}, x_{1}, \ldots, x_{n},-x_{0},-x_{n+1}, \ldots,-x_{n+i-1}, x_{n+i+1}, \ldots\right) .
$$


Note that for $k=1,2, \ldots, n, n+i+1, n+i+2, \ldots$, the $k$ coordinate is fixed. In order to define $h^{\prime}$ it is merely necessary to "fill in the gaps", for the various $i$, for $t$ between $1-2^{-i}$ and $1-2^{-i-1}$. Note that the coordinate formulas for $h^{\prime}\left(x, 1-2^{-i}, n\right)$ and $h^{\prime}\left(x, 1-2^{-i-1}, n\right)$ differ only in the original 0 th and $(n+i+1)$ th places. In these two places we have $x_{n+i}$ and $x_{n+i+1}$ in the first case and $x_{n+i+1}$ and $-x_{n+i}$ in the second case. Thus we may "rotate" the coordinate space $X_{0} \times X_{n+i+1}$ to change these two coordinates as required. Technically, since $X_{0} \times X_{n+i+1}$ is an open square, we first contract the square onto the circular disc $x_{0}^{2}+x_{n+i+1}^{2}<1$, rotate the disc clockwise by a quarter turn and then expand it to the open square. For each $t \in\left[1-2^{-i}, 1-2^{-i-1}\right]$, the 0 th and $(n+i+1)$ th coordinates of $h^{\prime}(x, t, n)$ are to be the induced combination of $x_{i+1}$ and $x_{n+i+1}$ obtained by linearly identifying $t$ with the appropriate stage of the rotation. For $t \in\left[1-2^{-i}, 1-2^{-i-1}\right]$ all other coordinates are those of $h^{\prime}\left(x, 1-2^{-i}, n\right)$.

Finally to obtain $h$ from $h^{\prime}$ for $0<t<1$ we scale down the 0 th coordinate by a factor of $(1-t)$. That is, define $\mu: X_{0}^{\infty} \times I \rightarrow X_{0}^{\infty}$ by $\mu(x, t)=\left([1-t] x_{0}, x_{1}, \ldots\right)$ and then define $h$ by $h(x, t, n)=\mu\left(h^{\prime}(x, t, n), t\right)$. Such an $h$ is clearly continuous.

With $h$ defined we can now define $H$. First, let $\phi$ be a map of $U$ onto $I$ such that $\phi(U \backslash W)=0, \phi(V)=1$, and $\phi(z)=\phi\left(\pi_{n}(z)\right)$ for each $z \in U$. The last condition makes $\phi$ a special kind of product map and such a $\phi$ may be defined by first defining a map $\phi^{\prime}$ on $\pi_{n}(U)$ such that $\phi^{\prime}\left(\pi_{n}(z)\right)=0$ for $z \in U \backslash W$ and $\phi^{\prime}\left(\pi_{n}(z)\right)=1$ for $z \in V$. Then define $\phi$ by $\phi=\phi^{\prime} \pi_{n}$.

We now define $H: s \times U \rightarrow s \times U$ as follows. For $p=\left(y_{1}, y_{2}, \ldots, z_{1}, z_{2}, \ldots\right)$ $\in s \times U$, recall that $p^{i}=\left(y_{i}, z_{(i, 1)}, z_{(i, 2)}, \ldots\right)$. Let $[H(p)]^{i}=h\left(p^{i}, \phi^{*}(p), n\right)$. It is easy to verify that the map $H$ is a homeomorphism of $s \times U$ onto the variable product $s \times{ }^{r} U$ where $r=1-\phi$ and furthermore $H \mid s \times(U \backslash W)$ is the identity.

4. Lemma B: The general case. For the proof of Lemma $B$ in the general case the sets $U, V$, and $W$ need not be as nice as those used above. In particular, the sets $U$ and $W$ might be infinite unions of sets of the form of $U$ in the special case where the values of $n$ increase without bound. Furthermore the closed set $V$ need not have any obvious product structure. Thus, instead of having a fixed $n$ we have to introduce a new variable into our isotopy function $h$ which will allow us to pick out an $n$ for a particular local product structure and to continuously vary this value as the local product structure changes. Hence, we want to define a map

$$
h: X_{0}^{\infty} \times I \times[1, \infty) \rightarrow X_{0}^{\infty}
$$

such that for each integer $n \in[1, \infty)$ and each $x=\left(x_{0}, x_{1}, \ldots\right) \in X_{0}^{\infty}$, as $t$ varies from 0 to $1, h(x, t, n)$ goes through the same motion as described in the special case above taking $x$ at time $t=1-2^{-i}$ to the point

$$
\left(2^{-i} x_{n+i}, x_{1}, \ldots, x_{n},-x_{0},-x_{n+1}, \ldots,-x_{n+i-1}, x_{n+i+1}, \ldots\right)
$$

and to $\left(0, x_{1}, \ldots, x_{n},-x_{0},-x_{n+1}, \ldots\right)$ at $t=1$. Also we specify that for any $n \leqq u<n+1$ and any $t \in I, h(x, t, u)$ has the same $x_{1}$ to $x_{n}$ coordinates as $x$ and that 
the coordinate in the 0 th place has been shrunk by a factor of $1-t$. Finally, for $h$ to be continuous at $t=1$ we specify for $n \leqq u<n+1$ that $h(x, 1, u)$ is the appropriate intermediate value between $h(x, 1, n)$ and $h(x, 1, n+1)$. These two points differ only in the $(n+1)$ th and $(n+2)$ th coordinate places. In these places we have $-x_{0}$ and $-x_{n+1}$ in the first case and $x_{n+1}$ and $-x_{0}$ in the second case and for the intermediate value $u$ we take the appropriate "rotation" on these two coordinates leaving all other coordinates the same.

In the rest of this section we will prove Lemma B except for the proofs of Lemmas $\mathrm{C}, \mathrm{E}$, and $\mathrm{F}$. The proof of Lemma $\mathrm{C}$ will be postponed to $\S 6$ and amounts to the construction of the map $h$ described above. Lemmas $\mathrm{E}$ and $\mathrm{F}$ will be proved in $\$ 5$ and will assert the existence of appropriate local product maps $\phi$ and $g$ that will provide the values for the variables $t$ and $u$, respectively, in the definition of the homeomorphism $H$. We are now ready to state

LEMMA C. There exists a map

$$
h: X_{0}^{\infty} \times I \times[1, \infty) \rightarrow X_{0}^{\infty}
$$

such that if $t \in I$ and $u \in[1, \infty)$ are fixed where $n \leqq u$, then the map $H: s \times s \rightarrow s \times s$ defined by $[H(p)]^{i}=h\left(p^{i}, t, u\right)$ for $p \in s \times s$ is a homeomorphism of $s \times s$ onto $s \times{ }^{r} s$ where (1) $r$ is the constant function $1-t$, (2) if $t=0$, then $H$ is the identity, and (3) $\pi_{n}^{*}=\pi_{n}^{*} H$.

We will now modify Lemma $\mathrm{C}$ by using local product maps $\phi$ and $g$ in place of the $t$ and $u$ respectively.

LEMma D. Let $W$ be any open subset of $s$, let $s \times{ }^{r} \mathrm{~W}$ be a variable product of $s$ by $W$ where $r_{0}(x)>0$ for each $x \in W$, and let $\left\{G_{i}\right\}$ be a star finite collection of $m_{i}$-basic open sets covering $W$. Let $\phi: W \rightarrow I$ and $g: W \rightarrow[1, \infty)$ be local product and local product indicator maps of $W$, respectively, with respect to the $G_{i}$ and $m_{i}$. There exists an onto homeomorphism

$$
H: s \times{ }^{r}{ }^{0} W \rightarrow s \times{ }^{r} W
$$

where (1) $r=(1-\phi) r_{0}$ and (2) $H \mid s \times{ }^{r_{0}} \phi^{-1}(0)$ is the identity.

Proof. Clearly the map $k$ from $s \times{ }^{r_{0}} W$ to $s \times W$ defined by $k(y, z)=\left(y r_{0}^{-1}(z), z\right)$ is an onto homeomorphism. Let $h$ be the map of Lemma $\mathrm{C}$ and define $H_{1}: s \times W$ $\rightarrow s \times s$ by $\left[H_{1}(p)\right]^{i}=h\left(p^{i}, \phi^{*}(p), g^{*}(p)\right)$ for $i>0$ and $p \in s \times W$. By condition (3) of Lemma $C$ together with the local product map properties with respect to the $G_{i}$ and $m_{i}$ we observe (1) that $H_{1}$ maps $s \times W$ onto $s \times{ }^{r_{1}} W$ where $r_{1}=1-\phi$ and (2) that $\phi^{*}=\phi^{*} H_{1}$ and $g^{*}=g^{*} H_{1}$. Property (1) follows since if $p \in s \times W$ where $n \leqq g^{*}(p)$, then $H_{1}$ carries the set $\left(\pi_{n}^{*}\right)^{-1} \pi_{n}^{*}(p)$ onto itself and (2) follows since $\phi^{*}(p)=\phi \pi(p)=\phi \pi_{n} \pi(p)=\phi \pi_{n}^{*}(p)=\phi \pi_{n}^{*} H_{1}(p)=\phi^{*} H_{1}(p)$ and similarly, for $g^{*}$. We now show that $H_{1}$ has a continuous inverse. 
Define $G$ from $s \times{ }^{r_{1}} W$ to $s \times W$ as follows. Let $b \in s \times{ }^{r_{1}} W$ and let $H_{b}: s \times W$ $\rightarrow s \times^{r_{b}} s$ be defined by

$$
\left[H_{b}(p)\right]^{i}=h\left(p^{i}, \phi^{*}(b), g^{*}(b)\right) \text { for } i>0 \text { and } p \in s \times W,
$$

where $r_{b}=1-\phi^{*}(b)$. Let $x=G(b)=H_{b}^{-1}(b)$. Since $\phi^{*}=\phi^{*} H_{1}$ and $g^{*}=g^{*} H_{1}$ we have $H_{1}(x)=b$. Hence, it is clear that $G$ is the inverse of $H_{1}$ and $G$ is continuous since $h, \phi$, and $g$ are. Thus, $H_{1}$ is a homeomorphism. We now observe that $H$ defined on $s \times{ }^{r_{0}} W$ by $k^{-1} H_{1} k$ is a homeomorphism onto $s \times{ }^{r} W$ where $r=(1-\phi) r_{0}$ and that condition (2) of Lemma D is clear.

The next lemma will guarantee us that the proper kind of $\phi$ function can be constructed.

Lemma E. Let $U$ be an open subset of $s$ and let $V \subset W \subset U$ and $A \subset U$ where $W$ is open and $V$ and $A$ are closed relative to $U$. There exists a countable star finite collection $\left\{G_{i}\right\}$ of $m_{i}$-basic open sets in $s$ whose union is $W \backslash A$ and a map $\phi: U \backslash A \rightarrow I$ such that $\phi(V \backslash A)=1, \phi((U \backslash W) \backslash A)=0$ and $\phi \mid W \backslash A$ is a local product map of $W \backslash A$ with respect to the $G_{i}$ and $m_{i}$.

Proof. (See §5.)

The next lemma asserts the existence of the proper kind of $g$ function. Assume the same hypothesis as in Lemma $\mathrm{E}$.

LEMMA F. The collections $\left\{G_{i}\right\}$ and $\left\{m_{i}\right\}$ of Lemma $\mathrm{E}$ can be chosen such that there exists a local product indicator map $g: W \backslash A \rightarrow[1, \infty)$ with respect to the $G_{i}$ and $m_{i}$ where $g$ is unbounded near $A$, that is, for any $x \in A \cap \mathrm{Cl}(W \backslash A)$ and any $n>0$, there is a neighborhood $B(x)$ such that $g \mid(W \backslash A) \cap B(x)>n$. (By $\mathrm{Cl} C$ we mean the closure of $C$.)

\section{Proof. (See §5.)}

We now restate Lemma $B$ and prove it on the basis of the apparatus we have set up.

LEMMA B. Let $U$ be an open subset of $s$, let $V \subset W \subset U$ where $W$ is open and $V$ is closed in $U$, and let $s \times{ }^{r_{0}} U$ be a variable product of $s$ by $U$. There exists a homeomorphism $H$ of $s \times{ }^{r}{ }_{0} U$ onto a variable product $s \times{ }^{r} U$ such that $(1) r \leqq r_{0},(2) r(V)=0$, and (3) $H \mid s \times{ }^{r_{0}}\left[(U \backslash W) \cup r_{0}^{-1}(0)\right]$ is the identity.

Proof. By Lemmas E and F take a star finite collection $\left\{G_{i}\right\}$ of $m_{i}$-basic open sets and maps $\phi$ and $g$ satisfying the conditions of the lemmas for the case where $A=r_{0}^{-1}(0)$.

Now let $H^{\prime}$ be the homeomorphism $H$ of Lemma D defined with respect to $W \backslash r_{0}^{-1}(0), \phi$, and $g$. Define $H$ on $s \times{ }^{r_{0}} U$ by extending $H^{\prime}$ to the rest of $s \times{ }^{r_{0}} U$ with the identity function. Thus, if this extension is continuous, then $H$ will be a homeomorphism of $s \times{ }^{r} 0 U$ onto $s \times{ }^{r} U$ where $r=(1-\phi) r_{0}$ on $W \backslash r_{0}^{-1}(0)$ and $r=r_{0}$ on $(U \backslash W) \cup r_{0}^{-1}(0)$. We now show that this extension is continuous. Since 
$\phi\left((U \backslash W) \backslash r_{0}^{-1}(0)\right)=0$, by condition (2) of Lemma D the identity map on $s \times r_{0}\left[(U \backslash W) \mid r_{0}^{-1}(0)\right]$ and $H \mid s \times\left(W \backslash r_{0}^{-1}(0)\right)$ are compatible. To show that these are compatible with the identity on $s \times{ }^{r}{ }_{0} r_{0}^{-1}(0)$ we will check the coordinate-wise continuity of $H$. The continuity of $r_{0}$ gives the continuity of $H$ on the first, or $s$, coordinate and $g$ becoming unbounded near $r_{0}^{-1}(0)$ yields the continuity of $H$ on the second, or $U$, coordinate. Since conditions 1 and 2 of the statement of Lemma $\mathrm{B}$ are clear the proof is complete.

5. Proofs of Lemmas $\mathbf{E}$ and $\mathbf{F}$. Before proving Lemmas $E$ and $F$ we shall need a definition and another lemma.

Let $E$ be a basic open set in $s$ and let $n=\min \{i: E$ is $i$-basic $\}$. Then $E=E_{1} \times \cdots$ $\times E_{n} \times \prod_{i>n} J_{i}^{0}$ where each $E_{i}$ is an open subinterval of $J_{i}^{0}$. We say that $E^{+}=$ $E_{1} \times \cdots \times E_{n} \times \prod_{i>n} J_{i}$ is the $n$-basic open set in $J^{\infty}$ associated with $E$. Note that $E=E^{+} \cap s$.

Lemma G. Let $U$ be an open subset of $s$ and let $V \subset W \subset U$ and $A \subset U$ where $W$ is open and $V$ and $A$ are closed in $U$. There exist countable collections $\left\{W_{i}\right\}_{i \geqq 0}$ and $\left\{G_{i}\right\}_{i>0}$ of open sets in $U$ such that

(1) $V \subset W_{0}, \mathrm{Cl} W_{i} \subset W_{i+1} \subset W$ for each $i \geqq 0$ and the union of the $W_{i}$ 's $W$.

(2) $G=\left\{G_{i}\right\}_{i}>0$ is a star finite collection of basic open sets in $s$ whose union is $W \backslash A$ such that for each $i, \mathrm{Cl} G_{i} \subset W \backslash A$ and iffor some $j, \mathrm{Cl} G_{j}$ intersects $\left(\mathrm{Cl} W_{i}\right) \backslash W_{i-1}$, then $\mathrm{Cl} G_{j} \subset W_{i+1} \mid \mathrm{Cl} W_{i-2}$.

Proof. We assert the existence of the $W_{i}$ on the basis of standard elementary techniques of point-set topology. If $B$ is a subset of $U$, let $B^{\prime}$ denote $B \backslash A$. Now, for each $x \in\left(\mathrm{Cl} W_{1}\right)^{\prime}$ let $G_{x}$ be a basic open set in $s$ contained in $W_{2}^{\prime}$. For $i>1$ and for each $x \in\left(\mathrm{Cl} W_{i}\right)^{\prime} \backslash W_{i-1}$ let $G_{x}$ be a basic open set in $s$ contained in $W_{i+1}^{\prime} \mid \mathrm{Cl} W_{i-2}$. Let $H$ be the collection of all the $G_{x}$. The space $s$ is naturally imbedded in $J^{\infty}$. For each $G_{x}$ let $G_{x}^{+}$be the basic open set in $J^{\infty}$ associated with $G_{x}$ and let $W^{+}$be the union of all such $G_{x}^{+}$. Note that $W \backslash A=W^{+} \cap s$. Let

$$
F=\left\{B \mid B \text { is basic open in } J^{\infty} \text { and for some } G_{x} \in H, \mathrm{Cl} B \subset G_{x}^{+}\right\}
$$

and let $\left\{M_{i}\right\}_{i \geqq 0}$ be a sequence of compact subsets of $J^{\infty}$ such that for each $i \geqq 0$, $M_{i} \subset M_{i+1}^{0}$ and $\bigcup_{i \geqq 0} M_{i}=W^{+}$. Cover $M_{1}$ with elements of $F$ whose closures are contained in $M_{2}^{0}$ and by compactness extract a finite subcover $F_{1}$. For $i>1$, cover $\mathrm{Cl}\left(M_{i} \backslash M_{i-1}\right)$ with elements of $F$ whose closures are contained in $M_{i+1}^{0} \backslash M_{i-2}$ and by compactness extract a finite subcover $F_{i}$. Thus, one can find a countable star finite open cover $G^{+}$of $W^{+}$with basic open sets in $J^{\infty}$. Now let $G$ be the collection of intersections of the elements of $G^{+}$and $s$. Note that the closure of each element of $G$ is contained in $W \backslash A$.

We are now ready for the

Proof of Lemma E. Let $\left\{W_{i}\right\}_{i \geqq 0}$ and $\left\{G_{i}\right\}_{i>0}$ be as in the conclusion of Lemma G. 
Let

$$
B_{1}=\left\{G_{i}: \mathrm{Cl} G_{i} \cap V \neq \varnothing\right\}
$$

and for $k>1$, let

$B_{k}=\left\{G_{i}: G_{i} \notin \bigcup_{q=1}^{k-1} B_{q}\right.$ and there exists $G_{j} \in B_{k-1}$ such that $\left.\left(\mathrm{Cl} G_{i}\right) \cap\left(\mathrm{Cl} G_{j}\right) \neq \varnothing\right\}$.

For each $G_{i}$, let $n\left(G_{i}\right)=\min \left\{n: G_{i}\right.$ is $n$-basic $\}$ and $m\left(G_{i}\right)=\max \left\{n\left(G_{j}\right): \mathrm{Cl} G_{i}\right.$ $\left.\cap \mathrm{Cl} G_{j} \neq \varnothing\right\}$. Now, let $\left\{G_{2, i}\right\}$ be an enumeration of $B_{2}$ and inductively pick $m_{2, i} \geqq m\left(G_{2, i}\right)$ such that $m_{2, i+1} \geqq m_{2, i}$. For $k>2$ let $\left\{G_{k, i}\right\}$ be an enumeration of $B_{k}$ and pick $m_{k, i} \geqq m\left(G_{k, i}\right)$ such that $m_{k, i+1} \geqq m_{k, i}$ and if $\mathrm{Cl} G_{k, i} \cap \mathrm{Cl} G_{k-1, j} \neq \varnothing$, then $m_{k, i} \geqq m_{k-1, j}$. Now, if $G_{i}=G_{k, j}$ for some $k$ and $j$, let $m_{i}=m_{k, j}$ and if $G_{i} \notin \bigcup_{j>1} B_{j}$, let $m_{i}=m\left(G_{i}\right)$.

Define $\phi$ to be 1 on the union of the closures of the elements of $B_{1}$ and extend $\phi$ to the union of the closures of the elements of $B_{2}$ by induction on the $i$ in $\left\{G_{2, i}\right\}$. The induction step is the same as the first step so we shall only give the induction step. For $i=k$, use the Tietze extension theorem to extend $\phi$ to $\mathrm{Cl} G_{2, k}$ such that if $x \in \mathrm{Cl} G_{2, k} \cap \mathrm{Cl} G_{3, j}$, for some $j$, then $\phi(x)=\frac{1}{2}$, and if $x \in G_{2, k}$ then $1 / 2 \leqq \phi(x) \leqq 1$ and $\phi(x)=\phi\left(\pi_{m_{2, k}}(x)\right)$. This is possible since you first do it on the finite dimensional cell $\pi_{m_{2, k}}\left(\mathrm{Cl} G_{2, k}\right)$ and then extend to all of $\mathrm{Cl} G_{2, k}$ using the product structure. Extend $\phi$ to the rest of $\bigcup_{k>1, i>0} \mathrm{Cl} G_{k, i}$ such that if $x \in \mathrm{Cl} G_{k, i} \cap \mathrm{Cl} G_{k+1, j}$, for some $j$, then $\phi(x)=2^{-k+1}$, and if $x \in \mathrm{Cl} G_{k, i}$, then $2^{-k+1} \leqq \phi(x) \leqq 2^{-k+2}$ and

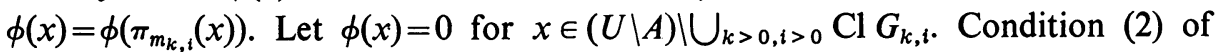
Lemma $\mathrm{G}$ guarantees, except for the case when $V=W=U$, that $\phi$ is continuous. If $V=U$, then $\phi$ is the constant function 1 . Thus, in any case we have satisfied the conditions of the lemma.

Proof of Lemma F. In the proof of the last lemma we could just as well have chosen the $m_{i}$ such that for each $i>0, m_{i+1} \geqq m_{i}$ and that the $m_{i}$ increase without bound. Assume this had been done. We now construct a local product indicator map $g: W \backslash A \rightarrow[1, \infty)$. For each $i>0$, let $C_{i}$ denote the closure of $G_{i}$ in $J^{\infty}$, recalling that $G_{i} \subset W \backslash A \subset s \subset J^{\infty}$. As a matter of convenience we shall define $g$ on the union of the $C_{i}$ and then restrict the function to $W \backslash A$ which is the common part of $s$ and this union.

For each $i>0$, let $r_{i}=$ maximum $\left\{m_{j}: C_{j} \cap C_{i} \neq \varnothing\right\}$. Define $g$ to be $r_{1}$ on $C_{1}$. We now extend to the rest of the $C_{i}$ by induction. Assume that $k>1$ is an integer and that $g$ has been extended to $\bigcup_{i=1}^{k-1} C_{i}$ such that if $x \in \bigcup_{i=1}^{k-1} C_{i}$ and $m_{j}=$ $\min \left\{m_{i}: x \in C_{i}, i=1, \ldots, k-1\right\}$, then $g(x)=g\left(\pi_{m_{j}}(x)\right)$ and $g(x) \geqq r_{j} \geqq m_{j}$. To extend $g$ to $C_{k}$ we have three cases: (1) $g$ has been defined on $C_{k}$, (2) $g$ has been defined for no point of $C_{k}$ and (3) $g$ has been defined for a proper nonvoid subset of $C_{k}$. If (1), proceed to $C_{k+1}$. If (2), define $g$ to be $r_{k}$ on $C_{k}$. If (3), proceed as follows. Let $H_{k}$ be the set of all $C_{i}$ that intersect $C_{k}$ and such that $g$ has been defined for no point of this intersection. Define $g$ to be $r_{k}$ on $C_{i} \cap C_{k}$, for each $C_{i} \in H_{k}$. Now take the set of all $C_{i}$ not in $H_{k}, i \geqq k$, that intersect $C_{k}$ and such that $g$ has been defined 
for some point of this intersection and let $D_{k}$ be the set of all intersections of $C_{k}$ with intersections of such $C_{i}$. Each element $B$ of $D_{k}$ is the intersection of a certain maximum number of the $C_{i}$; call this number the index of $B$. List the elements of $D_{k}$ as $B_{1}, \ldots, B_{n}$, according to nonincreasing index. It should be noted that $B_{n}=C_{k}$ and that, from the definition of the $m_{i}$, each $B_{i}$ is $m_{k}$-basic. We now extend $g$ inductively to the $B_{i}$. If $g$ has been defined on all of $B_{1}$, proceed to $B_{2}$. If $g$ has been defined for no point of $B_{1}$, let $g$ be equal to $r_{k}$ on $B_{1}$, and if $g$ has been defined on a proper nonvoid subset of $B_{1}$, extend $g$ as follows. Use the Tietze extension theorem to extend $g$ to $\pi_{m_{k}}\left(B_{1}\right)$ where the range of $g$ is the interval bounded by the maximum and minimum of the functional values assigned to points of $B_{1}$. Now, extend $g$ to the rest of $B_{1}$ using the product structure. Extend $g$ inductively to the rest of the $B_{i}$ which, at the last stage, includes $C_{k}$. The induction step is the same as the first step.

Thus, by induction we have extended $g$ to the union of the $C_{i}$. We now restrict $g$ to $W \backslash A$. By construction, $g$ is a local product indicator map with respect to the $G_{i}$ and $m_{i}$ and since the closure in $s$ of each $G_{i}$ is contained in $W \backslash A$ and since the $m_{i}$ are unbounded, then $g$ is unbounded near $A$.

6. Proof of Lemma C. The proof of Lemma C consists of constructing the map

$$
h: X_{0}^{\infty} \times I \times[1, \infty) \rightarrow X_{0}^{\infty}
$$

with the required properties. In $\S 3$ we have already described $h \mid X_{0}^{\infty} \times I \times\{n\}$ for $n \geqq 1$. Thus in defining $h$ our problem will be that of extending the function we already have to $h \mid X_{0}^{\infty} \times I \times[n-1, n]$ for $n \geqq 2$. Let us denote this restriction of $h$ by $h_{n}$. Following the pattern of $\S 3$ we shall first describe $h_{n}^{\prime}$ and then modify it to produce $h_{n}$. According to our description of $h^{\prime}$ in $\$ 3$ we have the following chart for $x=\left(x_{0}, x_{1}, \ldots\right) \in X_{0}^{\infty}, t \in I$ and $u \in[n-1, n]$ where $y$ denotes $\left(x_{1}, \ldots, x_{n-1}\right)$.

\begin{tabular}{r|r|r|r|r}
\multicolumn{1}{r}{$t$} & 0 & $1 / 2$ & \multicolumn{1}{c}{} \\
\hline$n$ & $x$ & $\begin{array}{r}\left(x_{n+1}, y, x_{n},-x_{0},\right. \\
\left.x_{n+2}, \ldots\right)\end{array}$ & $\begin{array}{r}\left(x_{n+2}, y, x_{n},-x_{0},\right. \\
\left.-x_{n+1}, x_{n+3}, \ldots\right)\end{array}$ & $\begin{array}{r}\left(x_{n+3}, y, x_{n},-x_{0},\right. \\
\left.-x_{n+1},-x_{n+2}, x_{n+4}, \ldots\right)\end{array}$ \\
\hline$n-1$ & $x$ & $\left(x_{n}, y,-x_{0}, x_{n+1}\right.$, & $\left(x_{n+1}, y,-x_{0},-x_{n}\right.$, & $\left(x_{n+2}, y,-x_{0},-x_{n}\right.$, \\
$\left.x_{n+2}, \ldots\right)$ & $\left.x_{n+2}, x_{n+3}, \ldots\right)$ & $\left.-x_{n+1}, x_{n+3}, x_{n+4}, \ldots\right)$ \\
\hline
\end{tabular}

FIGURE 1

We will describe three maps

(1) $h_{n, 0}^{\prime}: X_{0}^{\infty} \times[0,1 / 2] \times[n-1, n] \rightarrow X_{0}^{\infty}$,

(2) $h_{n, 1}^{\prime}: X_{0}^{\infty} \times[1 / 2,3 / 4] \times[n-1, n] \rightarrow X_{0}^{\infty}$ and

(3) $h_{n, 2}^{\prime}: X_{0}^{\infty} \times[3 / 4,7 / 8] \times[n-1, n] \rightarrow X_{0}^{\infty}$, 
and then show how to appropriately modify $h_{n, 2}^{\prime}$ to form

$$
h_{n, i}^{\prime}: X_{0}^{\infty} \times\left[1-2^{-i}, 1-2^{-i-1}\right] \times[n-1, n] \rightarrow X_{0}^{\infty}
$$

for $i>3$, where the $h_{n, j}^{\prime}$ patched together will yield $h_{n}^{\prime}$.

Description of $h_{n, 0}^{\prime}$. Let $\rho_{n}^{\prime}$ be the homeomorphism that takes $X_{0} \times X_{n} \times X_{n+1}$ onto the unit 3-ball $B_{n}=\left\{\left(x_{0}, x_{n}, x_{n+1}\right): x_{0}^{2}+x_{n}^{2}+x_{n+1}^{2}<1\right\}$ by shrinking linearly along radii. Let $\rho_{n}: X_{0}^{\infty} \rightarrow X_{0}^{\infty}$ be defined by $\rho_{n}=\rho_{n}^{\prime} \times$ id where id is the identity function of $\prod_{i \neq 0, n, n+1} X_{i}$. We now define a map

$$
h_{n, 0}^{\prime \prime}: B_{n} \times[0,1 / 2] \times[n-1, n] \rightarrow B_{n}
$$

as follows. Consider $t$ and $u$ to be elements of $[0,1 / 2]$ and $[n-1, n]$ respectively. For $t=0$ and $n-1 \leqq u \leqq n$ let $h_{n, 0}^{\prime \prime}$ be the identity. For $u=n-1$, as $t$ goes from 0 to $1 / 2$ let the $x_{0}$ and $x_{n}$ coordinates be rotated (using sine and cosine functions) so that $\left(x_{0}, x_{n}, x_{n+1}\right)$ is rotated to $\left(x_{n},-x_{0}, x_{n+1}\right)$. For $u=n$, as $t$ goes from 0 to $1 / 2$ let the $x_{0}$ and $x_{n+1}$ coordinates be rotated so that $\left(x_{0}, x_{n}, x_{n+1}\right)$ is rotated to $\left(x_{n+1}, x_{n},-x_{0}\right)$.

Fill in the rest of the isotopy as follows. For $n-1<u<n$ take a new axis $x_{u}$ that is proportionately between $x_{n}$ and $x_{n+1}$, see Figure 3 , and then rotate $x_{u}$ and $x_{0}$.

We will describe the resultant rotation for $t=1 / 2$ and as $u$ goes from $n-1$ to $n$. See Figure 2. This rotation is a double rotation, that is, starting with $\left(x_{n},-x_{0}\right.$,
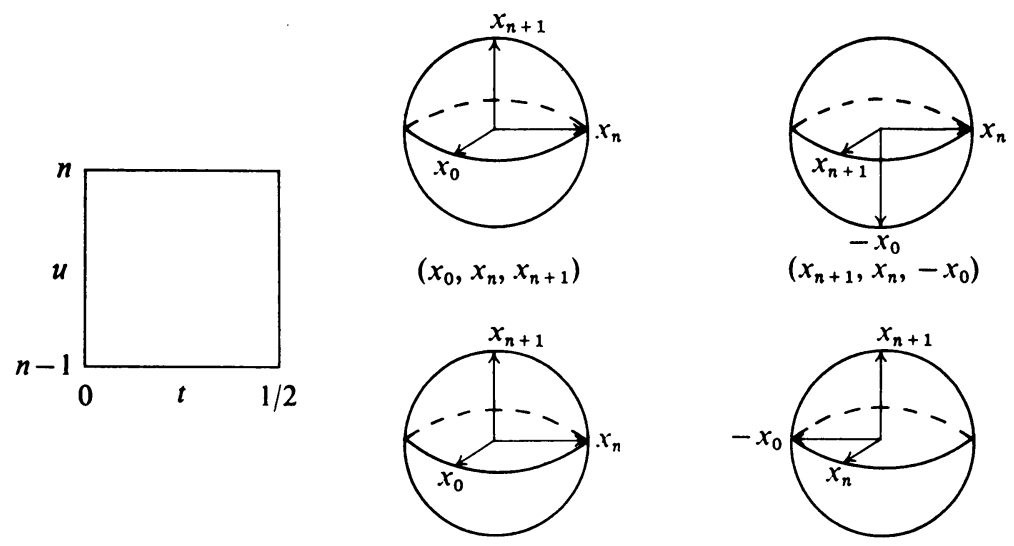

$\left(x_{0}, x_{n}, x_{n+1}\right)$

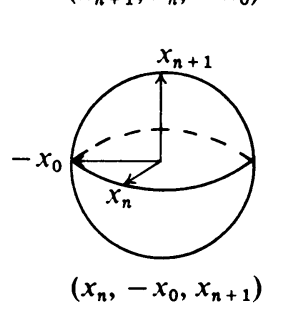

FIGURE 2

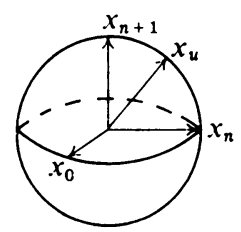

FIGURE 3 
$\left.x_{n+1}\right)$, as $x_{n+1}$ and $x_{n}$ are being rotated, so are $-x_{0}$ and $x_{n}$. Thus, $\left(x_{n},-x_{0}, x_{n+1}\right)$ ends up as $\left(x_{n+1}, x_{n},-x_{0}\right)$. It should be noted that an explicit formula for $h_{n, 0}^{\prime \prime}$ can be displayed as appropriate combinations of the sine and cosine functions.

Now, let $\hat{h}_{n, 0}=h_{n, 0}^{\prime \prime} \times$ id where id is the identity function of $\prod_{i \neq 0, n, n+1} X_{i}$. Then $h_{n, 0}^{\prime}=\rho_{n}^{-1} \hat{h}_{n, 0} \rho_{n}$.

Description of $h_{n, 1}^{\prime}$. Let $\sigma_{n}^{\prime}$ be the homeomorphism that takes $X_{0} \times X_{n} \times X_{n+1}$ $\times X_{n+2}$ onto the unit 4-ball

$$
C_{n}=\left\{\left(x_{0}, x_{n}, x_{n+1}, x_{n+2}\right): x_{0}^{2}+x_{n}^{2}+x_{n+1}^{2}+x_{n+2}^{2}<1\right\}
$$

by shrinking linearly along radii. Let $\sigma_{n}: X_{0}^{\infty} \rightarrow X_{0}^{\infty}$ be defined by $\sigma_{n}=\sigma_{n}^{\prime} \times$ id where id is the identity function of $\prod_{i \neq 0, n, n+1, n+2} X_{i}$. We now define

$$
h_{n, 1}^{\prime \prime}: C_{n} \times[1 / 2,3 / 4] \times[n-1, n] \rightarrow C_{n}
$$

as follows. For $x=\left(x_{0}, x_{n}, x_{n+1}, x_{n+2}\right) \in C_{n}$, let $h_{n, 1}^{n}(x, 1 / 2, n-1)=\left(x_{n},-x_{0}, x_{n+1}\right.$, $\left.x_{n+2}\right)$ and for $t=1 / 2$ as $u$ varies from $n-1$ to $n$ let $\left(x_{n},-x_{0}, x_{n+1}, x_{n+2}\right)$ go through the double rotation to $\left(x_{n+1}, x_{n},-x_{0}, x_{n+2}\right)$ as described in the definition of $h_{n, 0}^{n}$ (applied to the first three coordinates of $x$, keeping the $x_{n+2}$ coordinates fixed). See Figure 4. For $u=n-1$ and as $t$ goes from $1 / 2$ to $3 / 4$ let $x_{n}$ and $x_{n+1}$ be rotated

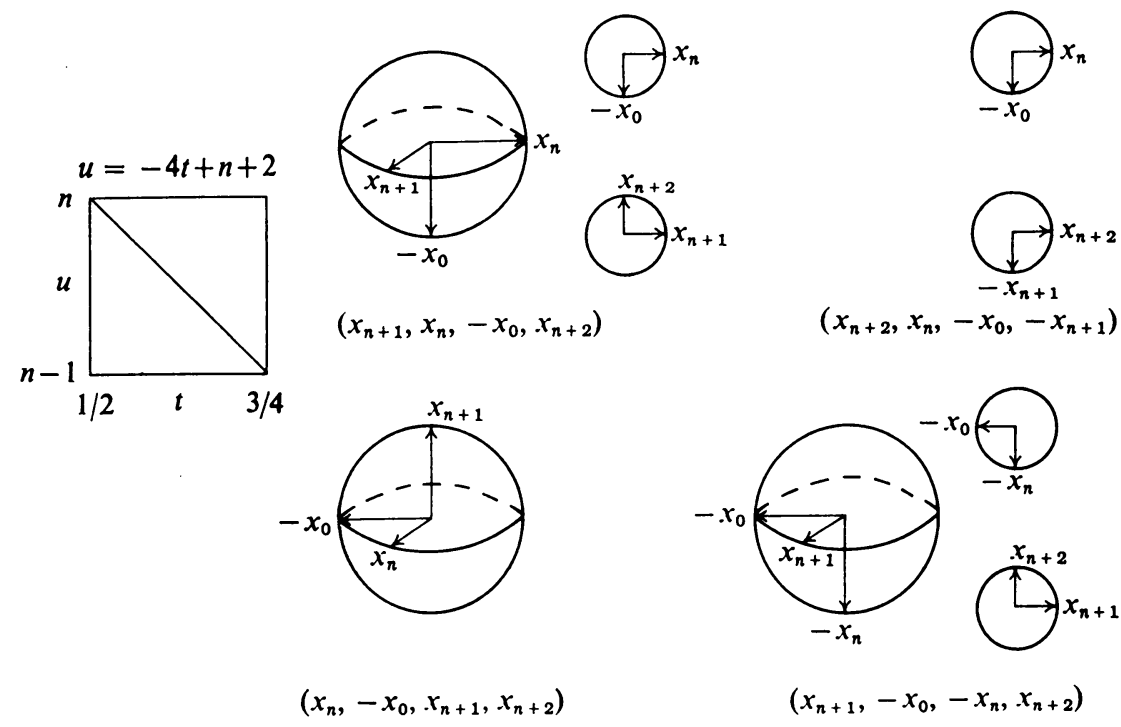

FIGURE 4

such that $\left(x_{n},-x_{0}, x_{n+1}, x_{n+2}\right)$ is rotated to $\left(x_{n+1},-x_{0},-x_{n}, x_{n+2}\right)$. We now describe a rotation corresponding to the diagonal $\{(t, u): 1 / 2 \leqq t \leqq 3 / 4, u=-4 t$ $+n+2\}$ of $[1 / 2,3 / 4] \times[n-1, n]$. As $(t, u)$, where $u=-4 t+n+2$, varies from 
$(1 / 2, n)$ to $(3 / 4, n-1)$, the $x_{n}$ and $-x_{0}$ coordinates are rotated so that $\left(x_{n+1}, x_{n}\right.$, $\left.-x_{0}, x_{n+2}\right)$ is rotated to $\left(x_{n+1},-x_{0},-x_{n}, x_{n+2}\right)$. Note that the double rotation corresponding to the path determined by $1 / 2 \times[n-1, n]$ is a combination of the rotations corresponding to the path determined by $[1 / 2,3 / 4] \times\{n-1\}$ and the diagonal. Hence, the map $h_{n, 1}^{\prime \prime}$ extends to the interior of the corresponding triangle. For $u=n$ as $t$ varies from $1 / 2$ to $3 / 4$, rotate the $x_{n+1}$ and $x_{n+2}$ coordinates so that $\left(x_{n+1}, x_{n},-x_{0}, x_{n+2}\right)$ rotates to $\left(x_{n+2}, x_{n},-x_{0},-x_{n+1}\right)$. For $t=3 / 4$ as $u$ varies from $n-1$ to $n$ we have a double rotation; $-x_{0}$ and $-x_{n}$ are rotated and inclependently but at the same time $-x_{n+1}$ and $x_{n+2}$ are rotated so that $\left(x_{n+1},-x_{0}\right.$, $\left.-x_{n}, x_{n+2}\right)$ is rotated to $\left(x_{n+2}, x_{n},-x_{0},-x_{n+1}\right)$. Note, for the triangle above the diagonal, that the rotations break up into pairs corresponding to Figure 5 . Hence
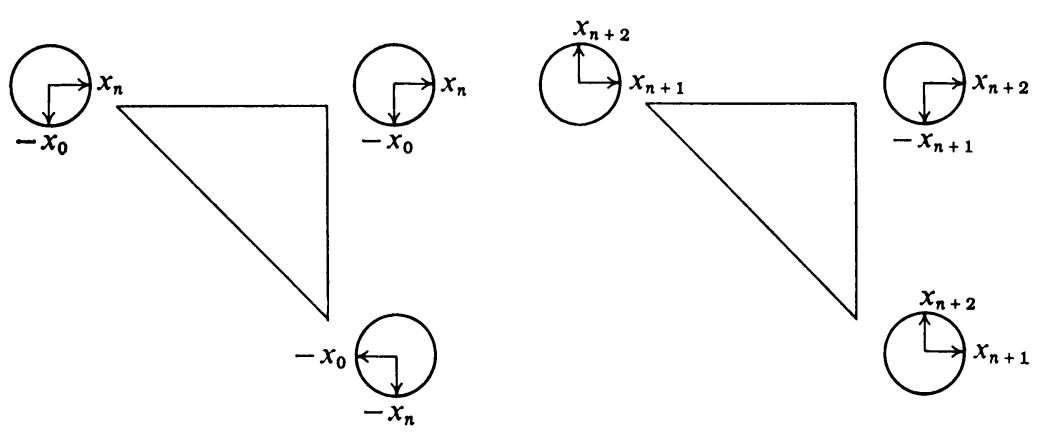

FIGURE 5

we can extend $h_{n, 1}^{\prime \prime}$ to this triangle and the definition of $h_{n, 1}^{\prime \prime}$ is complete. Now let $\hat{h}_{n, 1}=h_{n, 1}^{\prime \prime} \times$ id where id is the identity function of $\prod_{i \neq 0, n, n+1, n+2} X_{i}$. Then $h_{n, 1}^{\prime}=\sigma_{n}^{-1} \hat{h}_{n, 1} \sigma_{n}$.

Description of $h_{n, 2}^{\prime}$. Let $\tau_{n}^{\prime}$ be the homeomorphism of $X_{0} \times X_{n} \times X_{n+1} \times X_{n+2}$ $\times X_{n+3}$ onto

$$
D_{n}=\left\{\left(x_{0}, x_{n}, x_{n+1}, x_{n+2}, x_{n+3}\right): x_{n}^{2}+x_{n+1}^{2}<1 \text { and } x_{0}^{2}+x_{n+2}^{2}+x_{n+3}^{2}<1\right\}
$$

by shrinking linearly along radii. Note that $D_{n}$ is homeomorphic to the cartesian product of the 2-ball $E_{n}=\left\{\left(x_{n}, x_{n+1}\right): x_{n}^{2}+x_{n+1}^{2}<1\right\}$ and the 3-ball

$$
F_{n}=\left\{\left(x_{0}, x_{n+2}, x_{n+3}\right): x_{0}^{2}+x_{n+2}^{2}+x_{n+3}^{2}<1\right\} \text {. }
$$

At our convenience we may think of $D_{n}$ as it is defined or as $E_{n} \times F_{n}$. Let $\tau_{n}: X_{0}^{\infty} \rightarrow X_{0}^{\infty}$ be defined by $\tau_{n}=\tau_{n}^{\prime} \times$ id where id is the identity function on $\prod_{i \neq 0, n, n+1, n+2, n+3} X_{i}$. We now define a map

$$
h_{n, 2}^{\prime \prime}: D_{n} \times[3 / 4,7 / 8] \times[n-1, n] \rightarrow D_{n}
$$


as follows. For $x=\left(x_{0}, x_{n}, x_{n+1}, x_{n+2}, x_{n+3}\right) \in D_{n}$, let
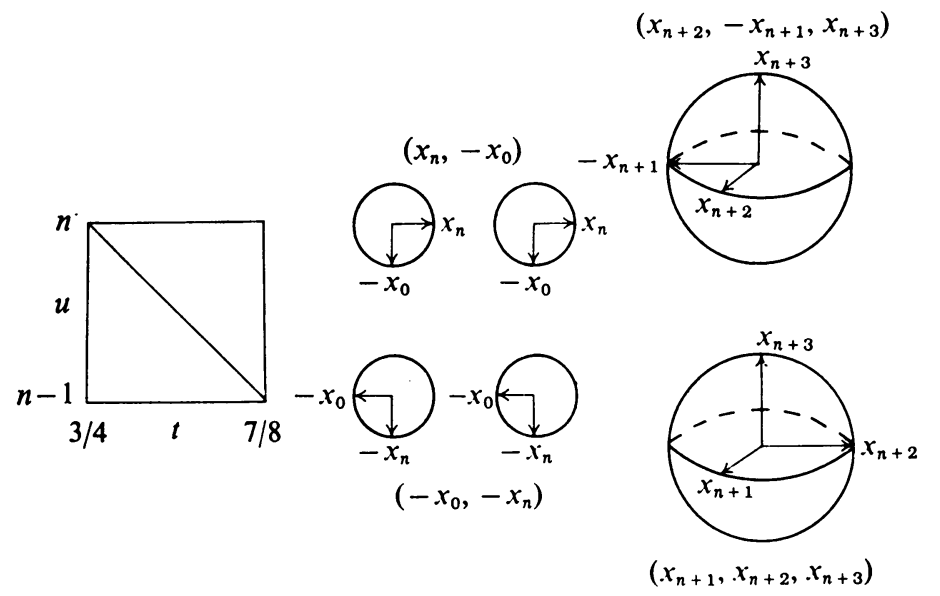
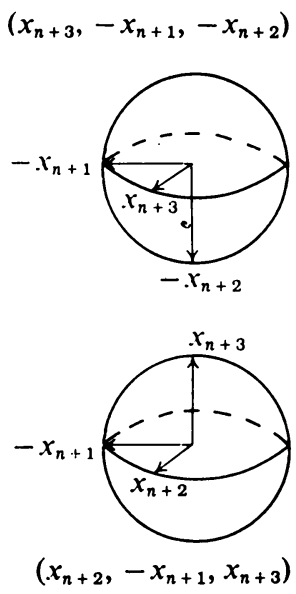

FIGURE 6

$h_{n, 2}^{\prime \prime}(x, 3 / 4,0)=\left(x_{n+1},-x_{0},-x_{n}, x_{n+2}, x_{n+3}\right)=\left(\left(-x_{0},-x_{n}\right),\left(x_{n+1}, x_{n+2}, x_{n, 3}\right)\right)$ and for $t=3 / 4$, as $u$ varies from $n-1$ to $n$, take the double rotation described in the definition of $h_{n, 1}^{\prime \prime}$. That is $\left(x_{n+1},-x_{0},-x_{n}, x_{n+2}, x_{n+3}\right)$ is rotated to $\left(x_{n+2}, x_{n},-x_{0},-x_{n+1}, x_{n+3}\right)$. Note that the rotations are done independently in the 2-ball and 3-ball respectively, and in the 3-ball the rotation is done only in the $x_{n+1}, x_{n+2}$-plane leaving the $x_{n+3}$ coordinate fixed. We now describe the rotations on the 2-ball. If $3 / 4 \leqq t \leqq 7 / 8$, as $u$ varies from $n-1$ to $n$ rotate $\left(-x_{0},-x_{n}\right)$ to $\left(x_{n},-x_{0}\right)$. That is, for each value of $t$, as $u$ varies from $n-1$ to $n$ we have the same rotation. We next describe the rotations on the 3-ball. For $u=n-1$, as $t$ varies from $3 / 4$ to $7 / 8$ we rotate $\left(x_{n+1}, x_{n+2}, x_{n+3}\right)$ to $\left(x_{n+2},-x_{n+1}, x_{n+3}\right)$. Note that this is the same rotation we have for $t=3 / 4$ as $u$ varies from $n-1$ to $n$. We now fill in the other two legs of the rectangle. That is, for $u=n$, as $t$ varies from $3 / 4$ to $7 / 8$ we have $\left(x_{n+2},-x_{n+1}, x_{n+3}\right)$ rotated to $\left(x_{n+3},-x_{n+1},-x_{n+2}\right)$ and for $t=3 / 4$, as $u$ varies from $n-1$ to $n$ we have the same rotation. Since both paths from $(3 / 4, n-1)$ to $(7 / 8, n)$ induce the same rotations, the map $h_{n, 2}^{\prime \prime}$ is extendible to the interior of the rectangle.

Let $\hat{h}_{n, 2}=h_{n, 2}^{n} \times$ id where id is the identity function on $\prod_{i \neq 0, n, n+1, n+2, n+3} X_{i}$. Then $h_{n, 2}^{\prime}=\tau_{n}^{-1} \hat{h}_{n, 1} \tau_{n}$.

We shall now describe what we mean by appropriately modified copies of $h_{n, 2}^{\prime}$ for the subintervals $\left[1-2^{-i}, 1-2^{-i-1}\right], i \geqq 3$. We describe the required rotation. For the $n$th and $(n+1)$ th coordinate places we want $\left(-x_{0},-x_{n}\right)$ rotated to $\left(x_{n},-x_{0}\right)$ as $u$ varies from $n-1$ to $n$. Otherwise we want to act on the 0 th, $(n+i)$ th and $(n+i+1)$ th coordinate places where the points

$$
\begin{aligned}
\left(x_{n+i},-x_{n+i-1}, x_{n+i+1}\right) & \left(x_{n+i+1},-x_{n+i-1},-x_{n+i}\right) \\
\left(x_{n+i-1}, x_{n+i}, x_{n+i+1}\right) & \left(x_{n+i},-x_{n+i-1}, x_{n+i+1}\right)
\end{aligned}
$$


correspond respectively to the lattice points of $\left[1-2^{-i}, 1-2^{-i-1}\right] \times[n-1, n]$. These rotations for the case $i=2$ are precisely what $h_{n, 2}^{\prime \prime}$ was designed to do. Indeed, in defining $h_{n, 2}^{\prime}$ we could just as well have defined, for $i>2$,

$$
h_{n, i}^{\prime}: X_{0}^{\infty} \times\left[1-2^{-i}, 1-2^{-i-1}\right] \times[n-1, n] \rightarrow X_{0}^{\infty} .
$$

It should be noted for $t=1-2^{-i}(i \geqq 3), x \in X_{0}^{\infty}$ and $n-1 \leqq u \leqq n$, that $h_{n, i-1}^{\prime}(x, t, u)$ $=h_{n, i}^{\prime}(x, t, u)$.

The map $h_{n}$. Let

$$
h_{n}^{\prime}: X_{0}^{\infty} \times[0,1) \times[n-1, n] \rightarrow X_{0}^{\infty}
$$

be the map obtained by patching together the $h_{n, j}^{\prime}$ for $j \geqq 0$. To scale down the 0 th coordinate we define $\mu: X_{0}^{\infty} \times I \rightarrow X_{0}^{\infty}$ as follows. For $x=\left(x_{0}, x_{1}, \ldots\right) \in X_{0}^{\infty}$ and $t \in I$, let $\mu(x, t)=\left([1-t] x_{0}, x_{1}, \ldots\right)$. From $h_{n}^{\prime}$ we define

$$
h_{n}: X_{0}^{\infty} \times I \times[n-1, n] \rightarrow X_{0}^{\infty}
$$

for $n>1$ as follows. For $x \in X_{0}^{\infty}, t \in[0,1)$, and $u \in[n-1, n]$, let $h_{n}(x, t, u)$ $=\mu\left(h_{n}^{\prime}(x, t, u), t\right)$ and for $t=1$, as $u$ varies from $n-1$ to $n$ let $\left(0, x_{1}, \ldots, x_{n-1}\right.$, $\left.-x_{0},-x_{n},-x_{n+1}, \ldots\right)$ be rotated to $\left(0, x_{1}, \ldots, x_{n-1}, x_{n},-x_{0},-x_{n+1}, \ldots\right)$. That is, in the $n$th and $(n+1)$ th coordinate places we have $\left(-x_{0},-x_{n}\right)$ rotated to $\left(x_{n},-x_{0}\right)$ leaving the other coordinates fixed. This yields a continuous $h_{n}$ since in the definition of $h_{n, i}^{\prime}$, for $i>1$, we used this same rotation on the $n$th and $(n+1)$ th coordinate places together with a rotation involving the 0 th, $(n+i)$ th, and $(n+i+1)$ th coordinates. The $1-t$ factor in the 0 th coordinate place and the fact that $i \rightarrow \infty$ as $t \rightarrow 1$ imply continuity for $h_{n}$.

Thus, $h_{n}$ is continuous and for $x \in X_{0}^{\infty}, t \in I$, and for $n>2$ we have $h_{n-1}(x, t, n-1)$ $=h_{n}(x, t, n-1)$. Hence we may patch the $h_{n}$ together to form

$$
h: X_{0}^{\infty} \times I \times[1, \infty) \rightarrow X_{0}^{\infty} .
$$

It is clear that if $t \in I$ and $u \in[1, \infty)$ where $n \leqq u$, then $h_{0}: X_{0}^{\infty} \rightarrow X_{0}^{\infty}$ defined by $h_{0}(x)=h(x, t, u)$ is a homeomorphism of $X_{0}^{\infty}$ onto $X_{0} \times X_{1}^{\infty}$ such that if $t=0$, then $h_{0}$ is the identity and that the $x_{1}$ to $x_{n}$ coordinates of a point $x \in X_{0}^{\infty}$ remain fixed. Hence, the map $H$ of Lemma $\mathrm{C}$ is defined coordinate-wise in terms of various copies of $h_{0}$ considered with respect to disjoint sets of coordinates and thus inherits the required properties.

ADDENDUM. In [7] David W. Henderson used Theorem I of this paper to prove that any $F$-manifold can be embedded as an open subset of $s$. With the aid of this result we obtain the following stronger version of Theorem I.

THEOREM. Let $M$ be any F-manifold and let $U$ be any open cover of $M$. There exists a homeomorphism $h: s \times M \rightarrow M$ such that for each $(x, y) \in s \times M$, there exists $u \in U$ such that $y$ and $h(x, y)$ are elements of $u$.

Proof. By [7] let $f$ be an open embedding of $M$ into $s$. Let $G$ be a countable star-finite collection of basic open sets in $s$ whose union is $f(M)$ such that $G$ 
refines $f(U)$. Let $g: f(M) \rightarrow[1, \infty]$ be a local product indicator map of $f(M)$ with respect to $G$. Consider the map $H$ of Lemma D defined with respect to $r_{0}=1$, $\phi=1$, and $g$. It follows that $H$ is a homeomorphism of $s \times f(M)$ onto $f(M)$ such that for each $(x, z) \in s \times f(M)$ we have $H(x, z)$ belonging to each element of $G$ that also contains $z$. Thus $h: s \times M \rightarrow M$ defined by $h=f^{-1} \circ H \circ(\mathrm{id} \times f)$ is the required homeomorphism.

\section{REFERENCES}

1. R. D. Anderson, Hilbert space in homeomorphic to the countable infinite product of lines, Bull. Amer. Math. Soc. 72 (1966), 515-519.

2. - Topological properties of the Hilbert cube and the infinite product of open intervals, Trans. Amer. Math. Soc. 126 (1967), 200-216.

3. R. D. Anderson, David W. Henderson and James E. West, Negligible subsets of infinitedimensional manifolds, Compositio Math. (to appear).

4. C. Bessaga, On topological classification of complete linear metric spaces, Fund. Math. 56 (1965), 251-288.

5. Czeslaw Bessaga and Victor Klee, Every non-normable Fréchet space is homeomorphic with all of its closed convex bodies, Math. Ann. 163 (1966), 161-166.

6. C. Bessaga and A. Pełeczyński, Some remarks on homeomorphisms of F-spaces, Bull. Acad. Polon. Sci. Sér. A Sci. Math. Astronom. Phys. 10 (1962), 265-270.

7. David W. Henderson, Infinite-dimensional manifolds are open subsets of Hilbert space, Topology (to appear).

8. M. I. Kadec, On topological equivalence of separable Banach spaces, Dokl. Akad. Nauk SSSR 167 (1966), 23-25 = Soviet Math. Dokl. 7 (1966), 319-322.

9. S. Kaplan, Homology properties of arbitrary subsets of euclidean spaces, Trans. Amer. Math. Soc. 62 (1947), 248-271

Louisiana State University, Baton Rouge, Louisiana 